\title{
Labyrinthe
}

$10 \mid 2001$

Numéro 10

\section{Un samedi après-midi dans une Gated Community}

Une image de sécession urbaine aux Ėtats-Unis

\section{Renaud Le Goix}

\section{CpenEdition}

Journals

Édition électronique

URL : http://journals.openedition.org/labyrinthe/1264

DOI : $10.4000 /$ labyrinthe. 1264

ISSN : 1950-6031

Éditeur

Hermann

Édition imprimée

Date de publication : 1 octobre 2001

Pagination : 85-94

Référence électronique

Renaud Le Goix, « Un samedi après-midi dans une Gated Community », Labyrinthe [En ligne], 10 | 2001, mis en ligne le 07 juin 2006, consulté le 19 avril 2019. URL : http://journals.openedition.org/ labyrinthe/1264; DOI : 10.4000/labyrinthe.1264

Ce document a été généré automatiquement le 19 avril 2019

Propriété intellectuelle 


\title{
Un samedi après-midi dans une Gated Community
}

Une image de sécession urbaine aux Ėtats-Unis

\author{
Renaud Le Goix
}

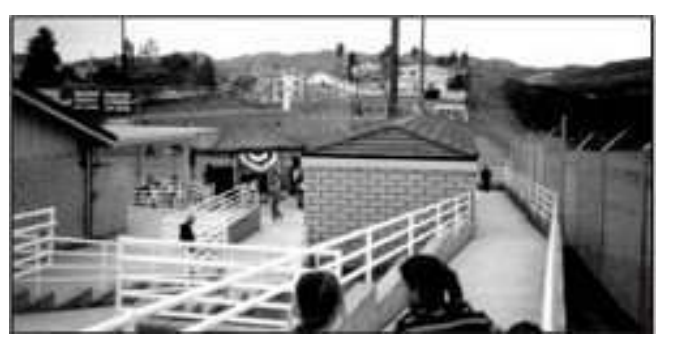

Autour d'un match de base-ball à Canyon Lake, Californie, décembre 1999 ; cliché R. Le Goix*

1 Le premier regard livre une image banale d'un samedi après-midi. Des enfants jouent au base-ball, dans des infrastructures de grande qualité. Les parents s'amusent aussi, fiers de leurs enfants, et heureux de se retrouver autour de cet événement local. On remarque les maisons individuelles à l'arrière-plan, belles, entretenues. Mais à droite de l'image, l'œil s'arrête sur les barbelés, la clôture, et la trace d'un chemin de ronde le long de celle-ci. Il semble séparer le quartier du désert, d'un no-man's land. Cette photographie aurait pu être prise dans l'Afrique du Sud de l'Apartheid, où townships et quartiers résidentiels étaient clôturés et fermés, mais on reconnaît ici les couleurs des États-Unis, accrochées sur un bâtiment. Il s'agit du Sud-Est de Los Angeles, là où le front d'urbanisation attaque les collines arides et caillouteuses du désert. Au-delà de la banalité du lotissement et de ses activités de loisirs, c'est la fermeture de celui-ci qui frappe. Ce quartier est en fait une véritable ville autonome : 15000 habitants, des services publics et de sécurité et des commerces à proximité, une municipalité autonome créée en 1990... et surtout une milice privée chargée de la sécurité et du contrôle des entrées et sorties.

2 Le terme de « gated community » désigne donc « des quartiers résidentiels dont l'accès est contrôlé, et dans lesquels l'espace public est privatisé. Leurs infrastructures de sécurité, 
généralement des murs ou grilles et une entrée gardée, protègent contre l'accès des nonrésidents. Il peut s'agir de nouveaux quartiers ou de zones plus anciennes qui se sont clôturées, et qui sont localisés dans les zones urbaines et périurbaines, dans les zones les plus riches comme les plus pauvres $»^{1}$. Au fond, bien plus que d'une réponse à la violence et à l'insécurité, il s'agit ici, par la privatisation et l'exclusivité des espaces publics (rues, parcs, lacs, plages...) d'ériger un critère de distinction entre ces quartiers et les immeubles plus traditionnels, contrôlés par un digicode ou surveillés par un concierge. Mode de périurbanisation, fondé sur la fermeture et sur une gestion privée par une association de copropriétaires, ces quartiers instituent un mode de vie qui contribue à l'accentuation des ségrégations et reflète à l'extrême la fragmentation sociale et politique des villes.

Ces phénomènes de fermeture résidentielle sont probablement issus de la tradition des beaux quartiers et ghettos dorés construits lors de la Révolution industrielle de la fin du XIX ${ }^{e}$ siècle dans les villes européennes ou sur la côte Est des États-Unis. Mais dans leur forme, ces développements contemporains sont plutôt inspirés par les quartiers fermés construits dans certains pays où les tensions intercommunautaires (sociales et/ou ethniques) sont particulièrement exacerbées, comme le Brésil, l'Argentine, l'Afrique du Sud.

4 Les premières clôtures résidentielles sont apparues aux États-Unis dans la seconde moitié du XIX $x^{e}$ siècle, dans les rues privées isolant les beaux quartiers de New York ou de SaintLouis. Les quartiers de Bel Air, à Beverly Hills, ou de Hidden Hills, ville privée abritant la jetset au nord-ouest de Los Angeles, représentent les héritiers californiens des ghettos dorés. Mais pour l'essentiel, les gated communities sont issues d'un modèle qui a émergé dans les années 1960, et qui s'est largement diffusé dans les années 1980-1990. S'il se développe dans l'ensemble des métropoles américaines, ce phénomène touche plus particulièrement les villes de la Sun Belt: Miami, Orlando, Houston, Dallas, Phoenix, Las Vegas, Los Angeles, où se trouve leur clientèle. En 1997, le nombre de gated communities aux États-Unis pouvait être estimé à 18000 . À Los Angeles, ces quartiers représentent plus de 200 lotissements de taille significative, soit plus de 250000 habitants, et plus de 10 $\%$ du marché immobilier du neuf.

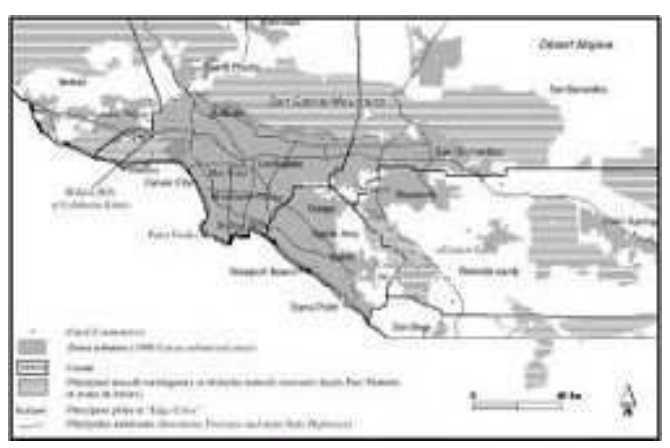

Sites et situations des gated communities dans l'aire urbaine de Los Angeles.

5 À Canyon Lake, la vie s'organise autour des activités de loisir : le base-ball et divers sports collectifs, mais aussi et surtout le golf ou les sports de glisse sur le petit lac de retenue qui irrigue le quartier. Le Country-Club constitue le pilier de la sociabilité locale, et organise les principaux événements et festivités. Partout, mer, golf, loisirs et soleil sont les piliers de ce type d'urbanisme fondé sur la qualité du mode de vie. Pour l'essentiel destinés aux classes moyennes et aisées, ces quartiers répondent à une recherche de communautés 
fondées sur un mode de vie et de loisirs (dites life-style communities) apportant une certaine tranquillité d'esprit, et une réponse à la peur de la violence urbaine. Il s'agit de quartiers périurbains à l'architecture et au mode de vie « communautaire » idéalisé, issus du marketing immobilier, comparables aux parcs à thème où ces communautés de retraités, de golfeurs ou d'amateurs de loisirs balnéaires vont chercher leurs références architecturales et urbanistiques. Comme tout produit immobilier, leur localisation met à profit un site plus ou moins prestigieux (une vallée, des collines, une plage) - dont l'accès se trouve ainsi privatisé -, et une situation pragmatique : le long des principales infrastructures qui permettent l'accès aux principaux centres d'activité de l'agglomération, ces edge-cities concentrent les activités de haute technologie, de la finance et les grands centres commerciaux.

Quartiers résidentiels certes, Canyon Lake - et les autres - se définissent avant tout comme des "communities", avec toutes les ambiguïtés que charrie le terme anglais. Les habitants décrivent la communauté comme l'expression d'un partage de valeurs, de respect et d'écoute des opinions de chacun. Ils la décrivent aussi comme un lieu privilégié de l'expression de la démocratie locale, comme un échelon de décision où chacun sait que son opinion peut compter. De façon générale, la communauté oscille donc entre une échelle politique, celle de la municipalité, et une échelle sociale de valeurs d'intérêts communs. L'essentiel est peut-être là. Le terme community recouvre en effet à la fois une signification identitaire (couleur, religion, niveau social...) et une signification politique, bien que la frontière, ou les liens entre les deux soient indécis. Faire partie de la même municipalité suppose une identité commune, un intérêt commun pour la gestion locale, et l'action politique se confond alors avec une démarche identitaire. La communauté, bâtie sur un consensus, est vue comme un moyen collectif d'action politique pour garantir des intérêts individuels.

7 Si la vie à Canyon Lake est pour l'essentiel guidée par un état d'esprit, un style de vie vendu comme plus glamour, d'autres communautés édictent des critères d'appartenance beaucoup plus rigoureux. Ainsi, Leisure World, à Orange County, au sud de Los Angeles, n'est autorisé qu'aux plus de 55 ans. Mais le critère a pu revêtir par le passé un caractère racial. Certains contrats de copropriété excluaient d'emblée les " non-caucasiens », c'està-dire tous ceux qui ne sont pas blancs d'origine européenne... Les lois anti-ségrégation ont rendu ces contrats caducs dans les années 1960, mais l'état d'esprit a manifestement perduré dans les faits. Le modèle de développement de ces quartiers est donc fondé sur un mode de gestion privé, par contrats de copropriété (dits Covenants, Conditions and Restrictions) et syndics (les Property Owners Associations), et l'homogénéité sociale voire ethnique est garante du consensus local.

8 Ces quartiers ne semblent pas si différents de l'ensemble des lotissements récents, aux États-Unis et ailleurs : socialement homogènes et gérés par un syndic. Mais la question de la clôture n'est pas anecdotique. Infrastructure essentielle, comme le montre la photo, celle-ci coûte cher. Un logement neuf dans une gated community vaut $10 \%$ de plus qu'ailleurs, à standing égal, et les frais de copropriété s'élèvent de 1000 à 1500 \$ par an, couvrant l'entretien de la clôture, des infrastructures de loisirs et des services collectifs et de sécurité. Mais ce coût n'est pas une simple commodité - un service -, mais bien la garantie de la pérennité de l'investissement immobilier. La comparaison des prix entre les gated communities et les quartiers environnants entre 1990 et 2000 montre nettement que l'investissement immobilier dans une gated community est mieux abrité des fluctuations conjoncturelles du marché. Ainsi, l'ensemble du marché immobilier à Los 
Angeles a été affecté par la crise immobilière liée entre autres aux émeutes de 1992, et aux tremblements de terre de 1993. Mais les gated communities ont mieux résisté à l'érosion des prix ; et depuis 1995-1996, leurs prix ont augmenté nettement plus vite que dans les quartiers environnants.

L'enclosure du quartier résidentiel renvoie ainsi à plusieurs niveaux d'interprétation. À la recherche de la sécurité et du bien-être des biens et des personnes, il faut ajouter l'exclusivité du lieu et du site, mais également la protection de la valeur de l'investissement immobilier: "Les propriétaires à Los Angeles, comme les Siciliens dans l'Honneur des Prizzi aiment leurs enfants, mais ils aiment encore plus la valeur de leur propriété $»^{2}$. Au-delà du niveau physique et social de l'enclosure au cœur des villes, les aspects juridiques, politiques et fiscaux de cette barrière révèlent une tendance à la sécession à peine dissimulée. Canyon Lake est ainsi plus qu'une simple association de copropriétaires : comme une ville médiévale, l'État de Californie lui a octroyé en 1990 une charte municipale. La confusion entre la sphère de la gestion privée (une copropriété exclusive) et les modalités de l'administration publique, produit des enclaves, fonctionnant en huis clos, extrêmement contrôlées voire militarisées. Sur la photo, la présence des barbelés trahit les tonalités martiales des gated communities. On n'y voit cependant pas les forces de sécurité privées, la milice autorisée par le second amendement de la Constitution des États-Unis, qui sont pourtant bien présentes à l'arrière-plan de la scène de divertissement familial.

10 Ce que ne montre pas la photo apparait pourtant en filigrane : il faut bien expliquer cette clôture qui fait violence au paysage. Ainsi, constater que l'immeuble qui abrite la municipalité de Canyon Lake est le même que celui de l'association de copropriétaires ouvre une piste. Il y a des précédents, et Canyon Lake n'est pas seule. Ainsi, à Los Angeles, quatre communautés ont obtenu leur indépendance municipale, la création de municipalités restant courante dans un pays neuf comme les États-Unis. L'incorporation est le processus par lequel une zone qui dépendait de l'administration du comté se voit octroyer le statut de municipalité, par une décision de l'État et par référendum local. Aux États-Unis en effet, il n'existe pas de maillage administratif systématique inférieur au comté: le statut de municipalité est donc une subdivision créée à l'occasion d'une demande expresse de municipalisation d'une zone agglomérée. Quatre gated communities sont ainsi devenues de véritables villes privées: Rolling Hills en 1957 (2 070 habitants), Hidden Hills en 1961 (1 850 habitants), Canyon Lake en 1990 (15000 habitants), Leisure World en 1999 ( 18000 habitants). Alors que les deux premières abritent une population très argentée et relèvent du ghetto doré, Canyon Lake et Leisure World (communauté réservée aux retraités, à deux pas de Disneyland...) sont des produits destinés aux classes moyennes. Ces villes sont essentiellement gérées par des associations de propriétaires, et l'action de la municipalité est réduite au strict minimum légal, se contentant de passer contrat avec le comté ou avec des entreprises privées pour les principaux services publics. Ce type de contrat est alors très avantageux pour les municipalités, du fait des économies d'échelle que réalise le comté pour ces services publics. Le zonage imposé par ces nouvelles municipalités les met également à l'abri de populations jugées indésirables. Elles échappent enfin - but ultime - à la fiscalité du comté, dont elles remettent en cause par nature le système de péréquation fiscale et de redistribution. L'attribution des permis de construire, l'organisation de la sécurité (contrôle d'accès et surveillance), l'entretien des infrastructures et les projets d'urbanisme sont gérés par la copropriété, donc par une entité privée. 
11 Et si ces incorporations de gated communities demeurent exceptionnelles, dans un certain nombre de cas non municipalisés, l'association de copropriétaires a su obtenir l'exemption d'une partie des frais de copropriété de la taxe foncière, garantissant ainsi une certaine autonomie de fonctionnement. Ainsi, les résidents de Woodbridge Village, à Irvine (au sud de Los Angeles) ont obtenu de pouvoir déduire de leurs taxes foncières les frais payés à l'association de copropriétaires pour l'entretien des routes, des espaces verts et la facturation des services de sécurité.

12 Cette confusion entre privé et public a déjà produit une jurisprudence intéressante. Il a ainsi été reproché à la municipalité de Leisure World d'interdire sur son sol la résidence des moins de 55 ans. Or, cette interdiction ne vaut que dans l'enceinte de la copropriété, et non dans la municipalité... la rhétorique peut prêter à rire, mais effectivement les -

pères fondateurs de ces villes ont soigneusement pris soin de laisser un espace municipal en dehors des murs, ne serait-ce que pour garantir un accès public à un hôtel de ville systématiquement situé à l'extérieur. Sur la photo, cette zone tampon ne mesure que quelques mètres, mais elle existe. Coto-de-Caza, non loin de Leisure World a quant à lui refusé à $80 \%$ des voix (lors d'un référendum local) l'implantation d'une école publique du comté dans ses murs. Destinée essentiellement aux enfants de la communauté, celle-ci aurait dû admettre néanmoins quelques enfants du dehors, remettant en cause l'exclusivité du site.

13 Parce qu'ils renforcent les effets des ségrégations socio-spatiales, ces quartiers posent d'autant plus de problèmes pour l'équilibre des métropoles qu'ils sont en quête d'indépendance politique ou d'exemptions fiscales. Quant à tous les quartiers fermés, qui plus traditionnellement n'ont ni exemption, ni indépendance, il s'agit souvent pour eux de se faire représenter - sous forme de lobbying des associations de copropriétaires notamment - dans les différentes instances publiques, au niveau du comté comme au niveau de la ville.

14 Mais au-delà du rôle des associations de copropriétaires, c'est le rôle des promoteurs, et en particulier de quelques firmes multi-nationales comme Kaufman \& Broad (par exemple) qu'il faut relever. Les quelques grandes gated communities dont il a été ici question - et en particulier Canyon Lake - sont considérées comme des modèles, des standards en matière de quartiers fermés. Ainsi, ces firmes commercialisent depuis une vingtaine d'années des lotissements fermés, souvent de moins de 500 habitants. Ces petites communautés constituent l'essentiel d'un marché de la promotion immobilière qui conçoit le logement comme un produit de grande consommation. Les prix s'échelonnent d'environ 90000 \$ à plus de 2 millions de dollars. On est loin des vraies villes privées comme Canyon Lake, mais les motivations sont les mêmes : sécurité, contrôle privé sur un site exclusif, protection $\mathrm{du}$ patrimoine... Enfin, par extension du modèle certains quartiers de public housing ont également été enclos, et font l'objet d'un contrôle d'accès. Mais il s'agit souvent là de politiques délibérées des autorités publiques des grandes villes (Los Angeles ou Atlanta par exemple).

Et l'extension du modèle, en particulier par le biais de grandes firmes multinationales, n'est pas qu'américaine. En Provence, Kaufman \& Broad a transformé en communauté fermée un ancien village de vacances à Pont Royal, près de Salon-de-Provence. En région parisienne, les petits lotissements fermés fleurissent, dans la vallée de la Bièvre, en vallée de Chevreuse, apportant dans les périphéries des modalités de fermeture que l'on ne connaissait que dans quelques beaux quartiers $\mathrm{du} 16^{\mathrm{e}}$ arrondissement. 
Quelques centaines de mètres de barbelés en disent long sur la privatisation des structures et des espaces résidentiels. La notion de «communauté » n'est souvent dans les faits que le vecteur d'un modèle immobilier garantissant la pérennité de l'investissement et la protection des intérêts individuels. Si la fermeture peut apparaître comme une contrainte, en particulier pour le visiteur, celle-ci est consentie dans l'intérêt bien compris de chacun. Mais la photographie ne peut montrer que les gated communities participent d'un phénomène plus général de privatisation de la gestion des espaces publics à l'échelle des métropoles (comme les centres commerciaux par exemple), et ne sont qu'un exemple de ce mode de "gouvernance" locale où la communauté consensuelle et le mode de vie par contrat de droit privé garantissent la protection des intérêts particuliers. Mais il ne faut pas trop en faire dire à des joueurs de base-ball, un samedi après-midi...

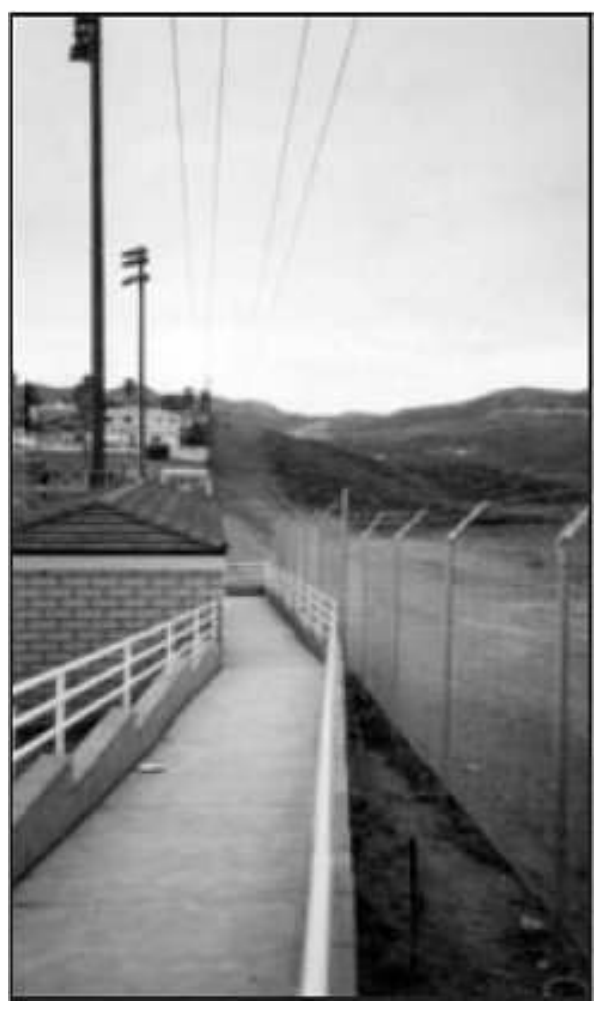

\section{NOTES}

*. Cette photographie est issue d'une thèse en cours de préparation : Les Gated Communities dans les métropoles des États-Unis, morceaux de villes ou territoires à part entière?, Université de Paris I-Panthéon-Sorbonne. Voir R. Le Goix (2001), « Les “communautés fermées" dans les villes des États-Unis. Aspects géographiques d'une sécession urbaine ", L'Espace géographique, 2001, vol. 30, nº 1, p. 81-93. 
1. E.J. Blakely, M.G. Snyder (1997), Fortress America, Gated Communities in the United States, Brookings Institution Press, Washington D. C., Lincoln Institute of Land Policy, Cambridge, M. A., 209 p.

2. M. Davis (1990), City of Quartz, Excavating the Future of Los Angeles, Londres, Verso. Éd. française : Paris, La Découverte, 1997, 393 p.

\section{RÉSUMÉS}

Quelques enfants jouent au base-ball un samedi après-midi, près de Los Angeles. La scène serait banale si elle n'avait pas lieu dans une "gated community", où un grillage de barbelés et des barrières protègent l'entrée du quartier contre les intrusions. Les gated communities sont des quartiers résidentiels enclos, protégés par des systèmes de sécurité et de contrôle d'accès, dont l'espace public est privatisé. Mais elles impliquent une fragmentation de la ville en communautés socialement homogènes (voire autosuffisantes pour un certain nombre de services), qui visent une certaine forme d'indépendance politique, véritable voie vers une sécession urbaine.

A Saturday afternoon in a gated community - What's happening on a Saturday afternoon in a gated community near Los Angeles? It could be assessed that gated communities make no differences: kids are just playing baseball anyway... But the baseball scene highly contrasts with the fence that surrounds and gates the neighborhood, protecting it against. « trespassers ». Gated Communities are walled residential neighborhoods with security and access control features, where the public space has been privatized. As a consequence, the whole city is parceled out into socially homogeneous and self-sufficient communities, which are looking for some kind of political independence, and may lead to real political secessions.

\section{AUTEUR}

\section{RENAUD LE GOIX}

Renaud Le Goix, né en 1973, ancien élève de l'ENS de Fontenay-Saint-Cloud, est agrégé de géographie. Allocataire-Moniteur à l'université Paris I-Panthéon-Sorbonne, il prépare actuellement une thèse sous la direction de Thérèse Saint-Julien sur Les Gated Communities dans les métropoles des États-Unis, morceaux de villes ou territoires à part entière? rlg(at)parisgeo.cnrs.fr 